\title{
On Some Maximal Galois Coverings over Affine and Projective Planes II
}

\author{
Tetsuo NAKANO and Hiroyasu NISHIKUBO
}

Tokyo Denki University

(Communicated by T. Nagano)

\section{Introduction.}

This note is the second part of the preceding paper [NT] which we refer as Part I in the following. We recall that in Part I the finite maximal Galois coverings over a complex affine plane $\mathbf{A}^{2}$ with branch locus $B_{q}:=\left\{(v, w) \in \mathbf{A}^{2} \mid w^{2}=v^{q}\right\}$ with $q$ odd were studied in some detail, and further, the existence of maximal Galois coverings over a complex projective plane $\mathbf{P}^{2}$ with branch locus $\overline{B_{q}} \cup l_{\infty}$ was discussed, where $\overline{B_{q}}$ is the projective closure of $B_{q}$ and $l_{\infty}$ is the infinite line.

In this note, we study the finite maximal Galois coverings over $\mathbf{A}^{2}$ (resp. $\mathbf{P}^{2}$ ) with branch locus $B_{q}$ (resp. $\overline{B_{q}} \cup l_{\infty}$ ) with even $q$, which remained untouched in Part I.

Our main results are: (i) the maximal Galois covering of $\mathbf{A}^{2}$ with branch locus $B_{q}$ exists and is isomorphic to $\mathbf{A}^{2}$ if the corresponding maximal Galois group is finite (Theorem 4) and (ii) a criterion for the existence of maximal Galois coverings over $\mathbf{P}^{2}$ with branch locus $\overline{B_{q}} \cup l_{\infty}$ (Theorem 7).

We note that the finite maximal Galois coverings of $\mathbf{P}^{2}$ thus obtained are all rational so that they will hopefully be good examples of rational normal projective surfaces which are finite maximal Galois coverings over $\mathbf{P}^{2}$ with simple branch loci and also with explicitly calculated Galois groups.

Our main results mentioned above are easy consequences of the explicit calculations of the maximal Galois groups with some simple presentation. We thus spent many pages on elementary combinatorial group-theoretic computation, which may be boring. The reason for our doing this is twofold: (i) these explicit descriptions of the maximal Galois groups are essentially used in our main results (ii) it will be convenient for the readers who are not accustomed to combinatorial group theory.

In order to calculate the order of finite groups with given presentation, we used Cayley/Magma system, which performs the Todd-Coxeter process on computers. 


\section{Maximal Galois coverings over $\mathbf{A}^{2}$ with branch locus $B_{q}$.}

We recall that the maximal Galois group $G(M, D)$ of the pair $(M, D)$, where $M$ is a complex manifold and $D=\sum_{i=1}^{s} e_{i} D_{i}\left(e_{i}>0, D_{i}\right.$ : irreducible component $)$ is an effective divisor on $M$, is defined as follows:

$$
G(M, D):=\pi_{1}\left(M-\operatorname{Supp}(D), P_{0}\right) / N\left\langle\gamma_{1}^{e_{1}}, \cdots, \gamma_{s}^{e_{s}}\right\rangle,
$$

where $\pi_{1}\left(M-\operatorname{Supp}(D), P_{0}\right)$ is the fundamental group of the complement of support of $D$ in $M$ with respect to a base point $P_{0}, \gamma_{i}$ is the homotopy class of a closed path which starts from $P_{0}$, goes around $D_{i}$ once, and returns to $P_{0}$, and $N\left\langle\gamma_{1}^{e_{1}}, \cdots, \gamma_{s}^{e_{s}}\right\rangle$ is the smallest normal subgroup which contains $\left\{\gamma_{1}^{e_{1}}, \cdots, \gamma_{s}^{e_{s}}\right\}$.

We note that $G(M, D)$ is a Galois group which controls the branched coverings over $M$ with branch locus at most at $D$. See [NT, Section 1] or [Na, Chapter 1] for the details on the theory of branched coverings.

Now we specialize to the situation where $M=\mathbf{A}^{2}$. As the branch locus $D$, we consider $B:=\left\{(v, w) \in \mathbf{A}^{2} \mid w^{2}=v^{q}\right\}$, where $q=2 r>0$ is a positive even integer. Decompose $B_{q}$ as $B_{q}=B_{q}^{1} \cup B_{q}^{2}$, where $B_{q}^{1}:=\left\{(v, w) \in \mathbf{A}^{2} \mid w=v^{r}\right\}, B_{q}^{2}:=\left\{(v, w) \in \mathbf{A}^{2} \mid w=-v^{r}\right\}$. Thus $B_{q}$ has two nonsingular branches which contact at the origin $r$-times if $r \geq 2$. In this section, we will first calculate the finite maximal Galois groups $G\left(\mathrm{~A}^{2}, e_{1} B_{q}^{1}+e_{2} B_{q}^{2}\right)$ explicitly. In the following, we set $G\left(e_{1}, e_{2} ; q\right):=G\left(\mathrm{~A}^{2}, e_{1} B_{q}^{1}+e_{2} B_{q}^{2}\right)$ for short.

PROPOSITION 1. $G\left(e_{1}, e_{2} ; q\right)$ has a following presentation:

$$
G\left(e_{1}, e_{2} ; q\right) \cong\left\langle a, b \mid a^{e_{1}}=b^{e_{2}}=1,(a b)^{r}=(b a)^{r}\right\rangle .
$$

ProOF. See [NT, Proposition 2.1].

PROPOSITION 2. Let $e_{1}, e_{2} \geq 2, q=2 r>0$ be integers. Then $G\left(e_{1}, e_{2} ; q\right)$ is a finite group if and only if $\left(e_{1}, e_{2}, q\right)=\left(m_{1}, m_{2}, 2\right),\left(2,2,2 n_{1}\right),\left(2, n_{2}, 4\right),(2,3,6),(3,3,4)$, $(2,3,8),(2,4,6),(3,4,4),(2,3,10),(2,5,6),(3,5,4)$, up to permutation in $\left(e_{1}, e_{2}\right)$. Here $m_{i}, n_{i} \geq 2$ are arbitrary integers.

PROOF. We show only if part first. Assume $q \geq 4$. We recall that the polyhedral group $P(x, y, z)$ is defined as $P(x, y, z):=\left\langle c, d \mid c^{x}=d^{y}=(c d)^{z}=1\right\rangle(x, y, z \geq 2)$. Since $P(x, y, z)$ is a homomorphic image of $G\left(e_{1}, e_{2} ; q\right)$, the only if part follows from the well-known finiteness condition for $P(x, y, z): P(x, y, z)$ is finite if and only if $(x, y, z)=$ $(2,2, z)$ where $z \geq 2,(2,3,3),(2,3,4),(2,3,5)$ up to permutation.

The if part follows from calculating orders of these groups explicitly by the Todd-Coxeter process.

In the following proposition, we use the notations below.

\section{NOTATION.}

(1) $D_{2 n}:=\left\langle x, y \mid x^{n}=y^{2}=1, y^{-1} x y=x^{-1}\right\rangle$ : the dihedral group of order $2 n$.

(2) $Q_{8}:=\left\langle x, y \mid x^{2}=y^{2}, x^{4}=1, y^{-1} x y=x^{-1}\right\rangle$ : the quaternion group of order 8 .

(3) $\mathbf{S L}\left(2, \mathbf{Z}_{n}\right):=$ the special linear group of degree 2 with coefficients in $\mathbf{Z}_{n}=\mathbf{Z} / n \mathbf{Z}$. 
(4) Given two groups $H, N$ with a homomorphism $\alpha: H \rightarrow \operatorname{Aut}(N)$, we denote by $H \bowtie<$ the semi-direct product of $H$ with $N$. The product is defined as $\left(h_{1}, n_{1}\right)\left(h_{2}, n_{2}\right):=$ $\left(h_{1} h_{2}, n_{1}^{h_{2}} n_{2}\right)$, where $h_{i} \in H, n_{i} \in N$ and $n_{1}^{h_{2}}=\alpha\left(h_{2}\right)\left(n_{1}\right)$.

Proposition 3. Suppose that $G\left(e_{1}, e_{2} ; q\right)$ is a finite group. Then we have the following isomorphisms. The description of the isomorphisms $\varphi_{i}$ and the actions $\alpha_{j}, \beta_{k}$ is given in the proof.

(0) $\varphi_{0}: G\left(m_{1}, m_{2} ; 2\right) \cong \mathbf{Z}_{m_{1}} \times \mathbf{Z}_{m_{2}}$.

(1) $\varphi_{1}: G\left(2,2 ; 2 n_{1}\right) \cong D_{4 n_{1}}$.

(2) $\varphi_{2}: G\left(2, n_{2} ; 4\right) \cong \mathbf{Z}_{2} \underset{\alpha_{2}}{\propto}\left(\mathbf{Z}_{n_{2}} \times \mathbf{Z}_{n_{2}}\right)$.

(3) $\varphi_{3}: G(2,3 ; 6) \cong \mathbf{Z}_{2} \triangleright_{\beta_{3}}<\left(\mathbf{Z}_{3} \triangleright_{\alpha_{3}}<Q_{8}\right)$.

(4) $\varphi_{4}: G(3,3 ; 4) \cong\left(\mathbf{Z}_{3} \times \mathbf{Z}_{3}\right) \triangleright_{\alpha_{4}}<Q_{8}$.

(5) $\varphi_{5}: G(2,3 ; 8) \cong \mathbf{Z}_{2} \triangleright_{\beta_{5}}<\left(\mathbf{Z}_{3} \triangleright_{\alpha_{5}}<\mathbf{S L}\left(2, \mathbf{Z}_{3}\right)\right)$.

(6) $\varphi_{6}: G(2,4 ; 6) \cong \mathbf{Z}_{2} \triangleright_{\beta_{6}}<\left(\mathbf{Z}_{4} \triangleright_{\alpha_{6}}<\mathbf{S L}\left(2, \mathbf{Z}_{3}\right)\right)$.

(7) $\varphi_{7}: G(3,4 ; 4) \cong \mathbf{Z}_{12} \underset{\alpha_{7}}{\curvearrowright} \mathbf{S L}\left(2, \mathbf{Z}_{3}\right)$.

(8) $\varphi_{8}: G(2,3 ; 10) \cong \mathbf{Z}_{6} \triangleright_{\alpha_{8}}<\mathbf{S L}\left(2, \mathbf{Z}_{5}\right)$.

(9) $\varphi_{9}: G(2,5 ; 6) \cong \mathbf{Z}_{10} \curvearrowright_{\alpha 9}<\mathbf{S L}\left(2, \mathbf{Z}_{5}\right)$.

(10) $\varphi_{10}: G(3,5 ; 4) \cong \mathbf{Z}_{15} \underset{\alpha_{10}}{\infty} \mathbf{S L}\left(2, \mathbf{Z}_{5}\right)$.

ProOF. In this proof, we write $G:=G\left(e_{1}, e_{2} ; q\right)$ for short.

(0), (1) These follow immediately from the presentation given in Proposition 1. The isomorphism $\varphi_{0}$ is given by

$$
\varphi_{0}(a)=(1,0), \quad \varphi_{0}(b)=(0,1),
$$

where $(1,0)$ means $\left(1 \bmod m_{1}, 0 \bmod m_{2}\right) \in \mathbf{Z}_{m_{1}} \times \mathbf{Z}_{m_{2}}$. The isomorphism $\varphi_{1}$ is given by

$$
\varphi_{1}(a)=y, \quad \varphi_{1}(b)=y^{-1} x .
$$

(2) We first form a semi-direct product $\mathbf{Z}_{2} \underset{\alpha_{2}}{\ltimes}\left(\mathbf{Z}_{n_{2}} \times \mathbf{Z}_{n_{2}}\right)$, where the action $\alpha_{2}: \mathbf{Z}_{2} \rightarrow$ $\operatorname{Aut}\left(\mathbf{Z}_{n_{2}} \times \mathbf{Z}_{n_{2}}\right)$ is defined by

$$
\alpha_{2}(1)((1,0)):=(1,-1), \quad \alpha_{2}(1)((0,1)):=(0,-1) .
$$

Define a map $\varphi_{2}: G \rightarrow \mathbf{Z}_{2} \underset{\alpha_{2}}{<}<\left(\mathbf{Z}_{n_{2}} \times \mathbf{Z}_{n_{2}}\right)$ by

$$
\varphi_{2}(a):=(1,(0,0)), \quad \varphi_{2}(b):=(0,(1,0)) .
$$

A direct calculation shows that $\operatorname{ord}(1,(0,0))=2, \operatorname{ord}(0,(1,0))=n_{2}$ and $(1,(0,0))^{2}=$ $(0,(1,0))^{2}$ so that the map $\varphi_{2}$ is a well-defined group homomorphism. Since a set $\{(1,(0,0))$, $(0,(0,1)),(0,(1,-1))\}=\left\{\varphi_{2}(a), \varphi_{2}(b), \varphi_{2}\left(a^{-1} b a\right)\right\}$ generates $\mathbf{Z}_{2} \underset{\alpha_{2}}{\prec}<\left(\mathbf{Z}_{n_{2}} \times \mathbf{Z}_{n_{2}}\right), \varphi_{2}$ is surjective.

In order to show $\varphi_{2}$ is an isomorphism, it is enough to show $\operatorname{ord}(G)=2 n_{2}^{2}$. 
We calculate ord $(G)$ after [J, Chapter 9]. Consider the following exact sequence

$$
1 \rightarrow G^{\prime} \rightarrow G \underset{f}{\rightarrow} G^{a b} \rightarrow 1,
$$

where $G^{\prime}$ is the commutator subgroup of $G, G^{a b}$ the abelianization of $G$ and $f$ the natural surjection. Since $G^{a b} \cong \mathbf{Z}_{2} \times \mathbf{Z}_{n_{2}}$, we may identify $f$ with $f: G \rightarrow \mathbf{Z}_{2} \times \mathbf{Z}_{n_{2}}, f(a)=(1,0)$, $f(b)=(0,1)$.

We take a Schreier transversal $U$ of $G^{\prime}$ in $G$ as follows:

$U:=\left\{1, b, b^{2}, \cdots, b^{n_{2}-3}, b^{n_{2}-2}, b^{n_{2}-1}, a, a b, a b^{2}, \cdots, a b^{n_{2}-3}, a b^{n_{2}-2}, a b^{n_{2}-1}\right\}$.

Then the $B \hat{R}$-table and $B \hat{S}$-table are given as follows:

$B \hat{R}$-TABLE

\begin{tabular}{|c|c|c|c|c|c|}
\hline & $\bar{a}$ & $\bar{b}$ & $a^{2}$ & $b^{n} 2$ & $a b a b a^{-1} b^{-1} a^{-1} b^{-1}$ \\
\hline 1 & 1 & 1 & $a^{2}$ & $b^{n} 2$ & $a b a b a^{-1} b^{-1} a^{-1} b^{-1}$ \\
\hline$b$ & $b a b^{-1} a^{-1}$ & 1 & $b a^{2} b^{-1}$ & $b^{n} 2$ & bababa $a^{-1} b^{-1} a^{-1} b^{-2}$ \\
\hline$b^{2}$ & $b^{2} a b^{-2} a^{-1}$ & 1 & $b^{2} a^{2} b^{-2}$ & $b^{n_{2}}$ & $b^{2} a b a b a^{-1} b^{-1} a^{-1} b^{-3}$ \\
\hline & : & : & & 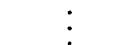 & \\
\hline$b^{n}{ }^{n-3}$ & $b^{n_{2}-3} a b^{-n_{2}+3} a^{-1}$ & 1 & $b^{n_{2}-3} a^{2} b^{-n_{2}+3}$ & $b^{n_{2}}$ & $b^{n} 2^{-3} a b a b a^{-1} b^{-1} a^{-1} b^{-n_{2}+2}$ \\
\hline$b^{n_{2}-2}$ & $b^{n_{2}-2} a b^{-n_{2}+2} a^{-1}$ & 1 & $b^{n_{2}-2} a^{2} b^{-n_{2}+2}$ & $b^{n} 2$ & $b^{n} 2^{-2} a b a b a^{-1} b^{-1} a^{-1} b^{-n_{2}+1}$ \\
\hline$b^{n_{2}-1}$ & $b^{n_{2}-1} a b^{-n_{2}+1} a^{-1}$ & $b^{n_{2}}$ & $b^{n_{2}-1} a^{2} b^{-n_{2}+1}$ & $b^{n} 2$ & $b^{n} 2^{-1} a b a b a^{-1} b^{-1} a^{-1} b^{-n_{2}}$ \\
\hline$a$ & $a^{2}$ & 1 & $a^{2}$ & $a b^{n} 2 a^{-1}$ & $a^{2} b a b a b a^{-1} b^{-1} a^{-1} b^{-1} a^{-1}$ \\
\hline$a b$ & $a b a b^{-1}$ & 1 & $a b a^{2} b^{-1} a^{-1}$ & $a b^{n} 2 a^{-1}$ & $a b a b a b a^{-1} b^{-1} a^{-1} b^{-2} a^{-1}$ \\
\hline$a b^{2}$ & $a b^{2} a b^{-2}$ & 1 & $a b^{2} a^{2} b^{-2} a^{-1}$ & $a b^{n} 2 a^{-1}$ & $a b^{2} a b a b a^{-1} b^{-1} a^{-1} b^{-3} a^{-1}$ \\
\hline • & & $\vdots$ & & $\vdots$ & \\
\hline$a b^{n_{2}-3}$ & $a b^{n_{2}-3} a b^{-n_{2}+3}$ & 1 & $a b^{n_{2}-3} a^{2} b^{-n_{2}+3} a^{-1}$ & $a b^{n} 2 a^{-1}$ & $a b^{n} 2^{-3} a b a b a^{-1} b^{-1} a^{-1} b^{-n_{2}+2} a^{-1}$ \\
\hline$a b^{n} 2^{-2}$ & $a b^{n_{2}-2} a b^{-n_{2}+2}$ & 1 & $a b^{n_{2}-2} a^{2} b^{-n_{2}+2} a^{-1}$ & $a b^{n} 2 a^{-1}$ & $a b^{n} 2^{-2} a b a b a^{-1} b^{-1} a^{-1} b^{-n_{2}+1} a^{-1}$ \\
\hline$a b^{n_{2}-1}$ & $a b^{n_{2}-1} a b^{-n_{2}+1}$ & $a b^{n} 2 a^{-1}$ & $a b^{n_{2}-1} a^{2} b^{-n_{2}+1} a^{-1}$ & $a b^{n} 2 a^{-1}$ & $a b^{n} 2^{-1} a b a b a^{-1} b^{-1} a^{-1} b^{-n} 2 a^{-1}$ \\
\hline
\end{tabular}

$B \hat{S}$-TABLE

\begin{tabular}{cc|ccc}
\hline- & - & $c_{n_{2}}$ & $c_{2 n_{2}}$ & $c_{n_{2}+1} c_{n_{2}+2}^{-1} c_{1}^{-1}$ \\
$c_{1}$ & - & $c_{1} c_{n_{2}+1}$ & $c_{2 n_{2}}$ & $c_{1} c_{n_{2}+2} c_{n_{2}+3}^{-1} c_{2}^{-1}$ \\
$c_{2}$ & - & $c_{2} c_{n_{2}+2}$ & $c_{2 n_{2}}$ & $c_{2} c_{n_{2}+3} c_{n_{2}+4}^{-1} c_{3}^{-1}$ \\
$\vdots$ & $\vdots$ & $\vdots$ & $\vdots$ & $\vdots$ \\
$c_{n_{2}-3}$ & - & $c_{n_{2}-3} c_{2 n_{2}-3}$ & $c_{2 n_{2}}$ & $c_{n_{2}-3} c_{2 n_{2}-2} c_{2 n_{2}-1}^{-1} c_{n_{2}-2}^{-1}$ \\
$c_{n_{2}-2}$ & - & $c_{n_{2}-2} c_{2 n_{2}-2}$ & $c_{2 n_{2}}$ & $c_{n_{2}-2} c_{2 n_{2}-1} c_{2 n_{2}} c_{n_{2}}^{-1} c_{2 n_{2}+1}^{-1} c_{n_{2}-1}^{-1}$ \\
$c_{n_{2}-1}$ & $c_{2 n_{2}}$ & $c_{n_{2}-1} c_{2 n_{2}-1}$ & $c_{2 n_{2}}$ & $c_{n_{2}-1} c_{2 n_{2}+1} c_{n_{2}} c_{n_{2}+1}^{-1} c_{2 n_{2}}^{-1}$ \\
$c_{n_{2}}$ & - & $c_{n_{2}}$ & $c_{2 n_{2}+1}$ & $c_{1} c_{2}^{-1} c_{n_{2}}^{-1}$ \\
$c_{n_{2}+1}$ & - & $c_{n_{2}+1} c_{1}$ & $c_{2 n_{2}+1}$ & $c_{n_{2}+1} c_{2} c_{3}^{-1} c_{n_{2}+2}^{-1}$ \\
$c_{n_{2}+2}$ & - & $c_{n_{2}+2} c_{2}$ & $c_{2 n_{2}+1}$ & $c_{n_{2}+2} c_{3} c_{4}^{-1} c_{n_{2}+3}^{-1}$ \\
$\vdots$ & $\vdots$ & $\vdots$ & $\vdots$ & $\vdots$ \\
$c_{2 n_{2}-3}$ & - & $c_{2 n_{2}-3} c_{n_{2}-3}$ & $c_{2 n_{2}+1}$ & $c_{2 n_{2}-3} c_{n_{2}-2} c_{n_{2}-1}^{-1} c_{2 n_{2}-2}^{-1}$ \\
$c_{2 n_{2}-2}$ & - & $c_{2 n_{2}-2} c_{n_{2}-2}$ & $c_{2 n_{2}+1}$ & $c_{2 n_{2}-2} c_{n_{2}-1} c_{2 n_{2}+1} c_{2 n_{2}}^{-1} c_{2 n_{2}-1}^{-1}$ \\
$c_{2 n_{2}-1}$ & $c_{2 n_{2}+1}$ & $c_{2 n_{2}-1} c_{n_{2}-1}$ & $c_{2 n_{2}+1}$ & $c_{2 n_{2}-1} c_{2 n_{2}} c_{1}^{-1} c_{n_{2}}^{-1} c_{2 n_{2}+1}^{-1}$ \\
\hline
\end{tabular}


Therefore we have

$$
\begin{aligned}
G^{\prime} & \left.\cong\left\langle c_{i}\left(1 \leq i \leq n_{2}\right)\right| 6 n_{2} \text { relations in the } B \hat{S} \text {-table }\right\rangle \\
& \cong\left\langle c_{1} \mid c_{1}^{n_{2}}=1\right\rangle \\
& \cong \mathbf{Z}_{n_{2}} .
\end{aligned}
$$

Hence $\operatorname{ord}(G)=\operatorname{ord}\left(G^{\prime}\right) \operatorname{ord}\left(G^{a b}\right)=2 n_{2}^{2}$ as desired.

(3) Consider a group $\mathbf{Z}_{2} \triangleright_{\beta_{3}}<\left(\mathbf{Z}_{3} \triangleright_{\alpha_{3}}<Q_{8}\right)$, where the actions $\beta_{3}, \alpha_{3}$ are defined as follows: The action $\alpha_{3}: \mathbf{Z}_{3} \rightarrow \operatorname{Aut}\left(Q_{8}\right)$ is defined by

$$
\alpha_{3}(1)(x):=y x^{2}, \quad \alpha_{3}(1)(y):=y x
$$

and the action $\beta_{3}: \mathbf{Z}_{3} \rightarrow \operatorname{Aut}\left(\mathbf{Z}_{3} \underset{\alpha_{3}}{\triangleright}<Q_{8}\right)$ is defined by

$$
\beta_{3}(1)((1,1)):=(1, y), \quad \beta_{3}(1)((0, x)):=\left(0, x^{3}\right), \quad \beta_{3}(1)((0, y)):=\left(0, y x^{2}\right) .
$$

Now define a map $\varphi_{3}: G \rightarrow \mathbf{Z}_{2} \triangleright_{\beta_{3}}<\left(\mathbf{Z}_{3} \bowtie_{\alpha_{3}}<Q_{8}\right)$ by

$$
\varphi_{3}(a):=(1,(0, e)), \quad \varphi_{3}(b):=(0,(1, e)),
$$

where $e \in Q_{8}$ is the identity element. We note that, since ord $((1,(0, e)))=2, \operatorname{ord}((0,(1, e)))$ $=3,\{(1,(0, e)) \cdot(0,(1, e))\}^{3}=\{(0,(1, e)) \cdot(1,(0, e))\}^{3}$ by a direct calculation, $\varphi_{3}$ is a welldefined group homomorphism.

The map $\varphi_{3}$ is surjective since a set $\{(1,(0, e)),(0,(1, e)),(0,(0, y)),(0,(0, y x))\}=$ $\left\{\varphi_{3}(a), \varphi_{3}(b), \varphi_{3}\left(b^{-1} a^{-1} b a\right), \varphi_{3}\left(b^{-2} a^{-1} b a b\right)\right\}$ generates $\mathbf{Z}_{2} \triangleright_{\beta_{3}}<\left(\mathbf{Z}_{3} \underset{\alpha_{3}}{{ }_{\alpha_{3}}}<Q_{8}\right)$.

On the other hand, we have ord $(G)=48$ by the Todd-Coxeter process performed with Cayly/Magma system. Since ord $\left(\mathbf{Z}_{2} \triangleright_{\beta_{3}}<\left(\mathbf{Z}_{3} \triangleright_{\alpha_{3}}<Q_{8}\right)\right)=48$, we conclude $\varphi_{3}$ is an isomorphism.

Since the proofs of (4)-(10) below are similar to that of (3), we only indicate explicitly the isomorphism $\varphi_{i}$ and the actions $\alpha_{j}, \beta_{k}$, omitting the details.

(4) The isomorphism $\varphi_{4}: G \cong\left(\mathbf{Z}_{3} \times \mathbf{Z}_{3}\right) \underset{\alpha_{4}}{\triangleright}<Q_{8}$ by

$$
\varphi_{4}(a):=((1,0), e), \quad \varphi_{4}(b):=((0,1), x y) .
$$

The action $\alpha_{4}: \mathbf{Z}_{3} \times \mathbf{Z}_{3} \rightarrow \operatorname{Aut}\left(Q_{8}\right)$ is given by $\alpha_{3}((1,0))(x):=y x, \quad \alpha_{3}((0,1))(x):=y, \quad \alpha_{3}((1,0))(y):=x, \quad \alpha_{3}((0,1))(y):=y x$.

(5) Set $X:=\left[\begin{array}{ll}1 & 1 \\ 2 & 0\end{array}\right], Y:=\left[\begin{array}{ll}2 & 0 \\ 1 & 2\end{array}\right] \in \mathbf{S L}\left(2, \mathbf{Z}_{3}\right)$.

The isomorphism $\varphi_{4}: G \cong \mathbf{Z}_{2}{ }_{\beta_{5}}<\left(\mathbf{Z}_{3} \underset{\alpha_{5}}{\triangleright}<\mathbf{S L}\left(2, \mathbf{Z}_{3}\right)\right)$ is given by

$$
\varphi_{5}(a):=\left(1,\left(0, I_{2}\right)\right), \quad \varphi_{5}(b):=\left(0,\left(1, I_{2}\right)\right),
$$

where $I_{2} \in \mathbf{S L}\left(2, \mathbf{Z}_{3}\right)$ is the identity matrix.

The action $\alpha_{5}: \mathbf{Z}_{3} \rightarrow \operatorname{Aut}\left(\mathbf{S L}\left(2, \mathbf{Z}_{3}\right)\right)$ is given by

$$
\alpha_{5}(1)(X):=Y^{-1}, \quad \alpha_{5}(1)(Y):=X Y^{-1} .
$$


The action $\beta_{5}: \mathbf{Z}_{3} \rightarrow \operatorname{Aut}\left(\mathbf{Z}_{3} \underset{\alpha_{5}}{\propto} \mathbf{S L}\left(2, \mathbf{Z}_{3}\right)\right)$ is given by $\beta_{5}(1)\left(\left(1, I_{2}\right)\right):=(1, Y), \quad \beta_{5}(1)((0, X)):=\left(0, X^{-1}\right), \quad \beta_{5}(1)((0, Y)):=\left(0, Y^{-1}\right)$.

(6) Set $X:=\left[\begin{array}{ll}2 & 1 \\ 2 & 0\end{array}\right], Y:=\left[\begin{array}{ll}2 & 0 \\ 1 & 2\end{array}\right] \in \mathbf{S L}\left(2, \mathbf{Z}_{3}\right)$.

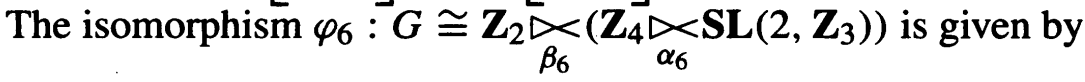

$$
\varphi_{6}(a):=\left(1,\left(0, I_{2}\right)\right), \quad \varphi_{6}(b):=\left(0,\left(1, I_{2}\right)\right) .
$$

The action $\alpha_{6}: \mathbf{Z}_{4} \rightarrow \operatorname{Aut}\left(\mathbf{S L}\left(2, \mathbf{Z}_{3}\right)\right)$ is given by

$$
\alpha_{6}(1)(X):=Y^{-1}, \quad \alpha_{6}(1)(Y):=Y X .
$$

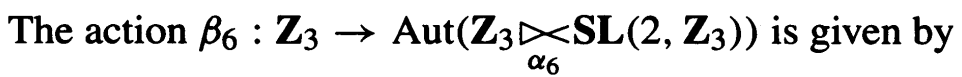
$\beta_{6}(1)\left(\left(1, I_{2}\right)\right):=(1, Y), \quad \beta_{6}(1)((0, X)):=\left(0, X^{-1}\right), \quad \beta_{6}(1)((0, Y)):=\left(0, Y^{-1}\right)$.

(7) Set $X:=\left[\begin{array}{ll}2 & 0 \\ 1 & 2\end{array}\right], Y:=\left[\begin{array}{ll}0 & 1 \\ 2 & 0\end{array}\right] \in \mathbf{S L}\left(2, \mathbf{Z}_{3}\right)$.

The isomorphism $\varphi_{7}: G \cong \mathbf{Z}_{12}{\underset{\alpha}{\alpha}}_{<} \mathbf{S L}\left(2, \mathbf{Z}_{3}\right)$ is given by

$$
\varphi_{7}(a):=\left(4, Y^{-1} X Y^{-1}\right)=\left(4,\left[\begin{array}{ll}
1 & 1 \\
0 & 1
\end{array}\right]\right), \quad \varphi_{7}(b):=\left(9, I_{2}\right) .
$$

The action $\alpha_{7}: \mathbf{Z}_{4} \rightarrow \operatorname{Aut}\left(\mathbf{S L}\left(2, \mathbf{Z}_{3}\right)\right)$ is given by

$$
\alpha_{7}(1)(X)=(X Y)^{-1}, \quad \alpha_{7}(1)(Y)=Y .
$$

(8) Set $X=\left[\begin{array}{ll}4 & 2 \\ 0 & 4\end{array}\right], Y=\left[\begin{array}{ll}4 & 0 \\ 2 & 4\end{array}\right] \in \mathbf{S L}\left(2, \mathbf{Z}_{5}\right)$.

The isomorphism $\varphi_{8}: G \cong \mathbf{Z}_{6} \triangleright_{\alpha_{8}}<\mathbf{S L}\left(2, \mathbf{Z}_{5}\right)$ is given by

$$
\varphi_{8}(a):=\left(3, I_{2}\right), \quad \varphi_{8}(b):=\left(4, Y^{2} X^{-2} Y^{-6}\right)=\left(4,\left[\begin{array}{ll}
4 & 4 \\
1 & 0
\end{array}\right]\right)
$$

The action $\alpha_{8}: \mathbf{Z}_{6} \rightarrow \operatorname{Aut}\left(\mathbf{S L}\left(2, \mathbf{Z}_{5}\right)\right)$ is given by

$$
\alpha_{8}(1)(X)=X^{-1}, \quad \alpha_{8}(1)(Y)=Y^{-1} .
$$

(9) Set $X=\left[\begin{array}{ll}4 & 2 \\ 0 & 4\end{array}\right], Y=\left[\begin{array}{ll}4 & 0 \\ 3 & 4\end{array}\right] \in \mathbf{S L}\left(2, \mathbf{Z}_{5}\right)$.

The isomorphism $\varphi_{9}: G \cong \mathbf{Z}_{10} \triangleright_{\alpha_{9}}<\mathbf{S L}\left(2, \mathbf{Z}_{5}\right)$ is given by

$$
\varphi_{9}(a):=\left(5, I_{2}\right), \quad \varphi_{10}(b):=\left(6, Y^{4} X^{-4} Y^{-4}\right)=\left(6,\left[\begin{array}{ll}
2 & 3 \\
3 & 0
\end{array}\right]\right)
$$

The action $\alpha_{9}: \mathbf{Z}_{10} \rightarrow \operatorname{Aut}\left(\mathbf{S L}\left(2, \mathbf{Z}_{5}\right)\right)$ is given by

$$
\alpha_{9}(1)(X)=X^{-1}, \quad \alpha_{9}(1)(Y)=Y^{-1} .
$$


(10) Set $X=\left[\begin{array}{ll}4 & 0 \\ 2 & 4\end{array}\right], Y=\left[\begin{array}{ll}1 & 3 \\ 3 & 0\end{array}\right] \in \mathbf{S L}\left(2, \mathbf{Z}_{5}\right)$.

The isomorphism $\varphi_{10}: G \cong \mathbf{Z}_{15} \underset{\alpha_{10}}{\triangleright<} \mathbf{S L}\left(2, \mathbf{Z}_{5}\right)$ is given by

$$
\varphi_{10}(a):=\left(10, Y^{2} X Y^{-1} X^{2}\right)=\left(10,\left[\begin{array}{ll}
3 & 4 \\
3 & 1
\end{array}\right]\right), \quad \varphi_{10}(b):=\left(6, I_{2}\right) .
$$

The action $\alpha_{10}: \mathbf{Z}_{10} \rightarrow \operatorname{Aut}\left(\mathbf{S L}\left(2, \mathbf{Z}_{5}\right)\right)$ is given by

$$
\alpha_{10}(1)(X)=X Y^{-1} X=\left[\begin{array}{ll}
1 & 2 \\
3 & 2
\end{array}\right], \quad \alpha_{10}(1)(Y)=X^{2} Y^{-1} X=\left[\begin{array}{ll}
4 & 3 \\
4 & 2
\end{array}\right] \text {. }
$$

Now we are ready for deducing a geometric result from Proposition 3.

Let $(M, D)$ be a pair of a complex manifold and an effective divisor on $M$. We recall that a Galois covering over $M$ which branches at $D$ is called the maximal Galois covering of $(M, D)$ if it is universal among the branched coverings over $M$ with branch locus at most at $D$ (cf. [NT, Definition 1.2]). Our first main result is the following:

THEOREM 4. For $\left(e_{1}, e_{2}, q\right)$ in Proposition 2, the maximal Galois covering of $\left(\mathbf{A}^{2}\right.$, $\left.e_{1} B_{q}^{1}+e_{2} B_{q}^{2}\right)$ exists and is all isomorphic to $\mathbf{A}^{2}$.

Proof. The existence of the maximal Galois covering $X$ follows from Proposition 3 and [NT, Corollary 1.5] by calculating explicitly the orders of $a$ and $b$ in $G\left(e_{1}, e_{2} ; q\right)$.

We show $X \cong \mathbf{A}^{2}$ in the case of $\left(e_{1}, e_{2}, q\right)=(3,5,4)$. The other cases are checked similarly. Let $H \subset \mathbf{S L}(2, \mathbf{C})$ be a binary icosahedral group, which is isomorphic to $\mathbf{S L}\left(2, \mathbf{Z}_{5}\right)$, and $f: \mathbf{A}^{2} \rightarrow \mathbf{A}^{2} / H \cong\left\{(x, y, z) \in \mathbf{C}^{3} \mid x^{2}+y^{3}+z^{5}=0\right\}$ the quotient map. By [NT, Proposition 2.3], we have a $\mathbf{Z}_{15}$-cyclic covering $g: \mathbf{A}^{2} / H \cong\left\{\left(u_{1}, u_{2}, v\right) \in \mathbf{C}^{3} \mid u_{1}^{3}+2 v^{2}-\right.$ $\left.u_{2}^{5}=0\right\} \rightarrow \mathbf{A}^{2},\left(u_{1}, u_{2}, v\right) \mapsto\left(v, u_{1}^{3}+v^{2}\right)$. Then we get a composite covering $g \circ f: \mathbf{A}^{2} \rightarrow$ $\mathbf{A}^{2}$ which branches at $3 B_{q}^{1}+5 B_{q}^{2}$.

Let $\pi: X \rightarrow \mathbf{A}^{2}$ be the maximal Galois covering of $\left(\mathbf{A}^{2}, 3 B_{q}^{1}+5 B_{q}^{2}\right)$. Then, by maximality, $g \circ f$ factors through $\pi$. Since the covering degrees of $g \circ f$ and $\pi$ are equal, we conclude that $g \circ f \cong \pi$.

REMARK. (1) In Part 1 [NT, Remark 2.7], we showed that also for $o d d q$ the maximal Galois covering of $\left(\mathbf{A}^{2}, e B_{q}\right)$ exists and is all isomorphic to $\mathbf{A}^{2}$ if the corresponding maximal Galois group is finite.

(2) The finite maximal Galois groups in Proposition 3, when considered as subgroups of $\mathbf{G L}(2, \mathbf{C})$, are all complex reflection groups (cf. [ST]). Indeed, by Theorem 4, we have $\mathbf{A}^{2} / G \cong \mathbf{A}^{2}$ where $G:=G\left(e_{1}, e_{2} ; q\right)$. Since $G$ is a finite group, we may assume $G \subset$ GL $(2, \mathbf{C})$ by a polynomial change of coordinates (cf. $[\mathrm{K}])$. Hence $G$ is a complex reflection group by [ST, 5.1]. 
3. Maximal Galois coverings over $\mathbf{P}^{2}$ with branch locus $\overline{B_{q}} \cup l_{\infty}$.

In this section, we give a criterion for the existence of the maximal Galois coverings over $\mathbf{P}^{2}$ with branch locus $\overline{B_{q}^{1}} \cup \overline{B_{q}^{2}} \cup l_{\infty}$, where $\overline{B_{q}^{i}}$ is the projective closure of $B_{q}^{i}$ and $l_{\infty}$ the infinite line. We recall the following proposition in Part I.

Proposition 5. Let $q:=2 r>0$ be an even positive integer. Set $\overline{B_{q}}:=\left\{\left(x_{0}: x_{1}\right.\right.$ : $\left.\left.x_{2}\right) \in \mathbf{P}^{2} \mid x_{0}^{q}=x_{1}^{2} x_{2}^{q-2}\right\}=\overline{B_{q}^{1}} \cup \overline{B_{q}^{2}}$, where $\overline{B_{q}^{1}}:=\left\{\left(x_{0}: x_{1}: x_{2}\right) \in \mathbf{P}^{2} \mid x_{0}^{r}=x_{1} x_{2}^{r-1}\right\}$, $\overline{B_{q}^{2}}:=\left\{\left(x_{0}: x_{1}: x_{2}\right) \in \mathbf{P}^{2} \mid x_{0}^{r}=-x_{1} x_{2}^{r-1}\right\}$ and $l_{\infty}:=\left\{\left(x_{0}: x_{1}: x_{2}\right) \in \mathbf{P}^{2} \mid x_{2}=0\right\}$ the infinite line. Then the presentation of the maximal Galois group $G\left(\mathbf{P}^{2}, e_{1} \overline{B_{q}^{1}}+e_{2} \overline{B_{q}^{2}}+m l_{\infty}\right)$ is given as follows:

$$
G\left(\mathbf{P}^{2}, e_{1} \overline{B_{q}^{1}}+e_{2} \overline{B_{q}^{2}}+m l_{\infty}\right) \cong\left\langle a, b, c \mid a^{e_{1}}=b^{e_{2}}=c^{m}=1,(a b)^{r}=(b a)^{r}=c^{-1}\right\rangle .
$$

PROOF. See [NT, Section 3].

Unfortunately, we do not know precisely when $G\left(\mathbf{P}^{2}, e_{1} \overline{B_{q}^{1}}+e_{2} \overline{B_{q}^{2}}+m l_{\infty}\right)$ is finite. We therefore restrict ourselves to the easiest cases where this group is clearly finite, namely, the cases where $G\left(\mathbf{P}^{2}, e_{1} \overline{B_{q}^{1}}+e_{2} \overline{B_{q}^{2}}+m l_{\infty}\right)$ is a quotient of the finite group $G\left(\mathbf{A}^{2}, e_{1} B_{q}^{1}+e_{2} B_{q}^{2}\right)$ in Proposition 3.

In the following, we also assume $q \geq 4$ so that we exclude the case (0) in Proposition 3, since we are mainly interested in non-abelian maximal Galois groups. We set $G\left[e_{1}, e_{2}, m ; q\right]:=G\left(\mathbf{P}^{2}, e_{1} \overline{B_{q}^{1}}+e_{2} \overline{B_{q}^{2}}+m l_{\infty}\right)$ for short.

PROPOSITION 6. Suppose that $G\left(e_{1}, e_{2} ; q\right)(q \geq 4)$ is a finite group (so that $\left(e_{1}, e_{2}, q\right)$ is one of the triples in Proposition 3 except the case (0)). Then the explicit structure of $G\left[e_{1}, e_{2}, m ; q\right]$ is given as follows:

1. $G\left[2,2, m ; 2 n_{1}\right] \cong \begin{cases}D_{2 n_{1}} & \text { if } m: \text { odd } \\ G\left(2,2 ; 2 n_{1}\right) & \text { if } m: \text { even }\end{cases}$

2. $G\left[2, n_{2}, m ; 4\right] \cong \mathbf{Z}_{2} \underset{\alpha_{21}}{\triangleright}\left(\mathbf{Z}_{l} \times \mathbf{Z}_{n_{2}}\right) \quad\left(l:=\operatorname{GCD}\left(m, n_{2}\right)\right)$

3. $G[2,3, m ; 6] \cong\left\{\begin{array}{lll}A_{4} & \text { if } m \equiv 1,3 & (\bmod 4) \\ \mathbf{Z}_{2} \underset{\beta_{31}}{\Delta}\left(\mathbf{Z}_{3} \underset{\alpha_{31}}{\triangleright}<\left(\mathbf{Z}_{2} \times \mathbf{Z}_{2}\right)\right) & \text { if } m \equiv 2 & (\bmod 4) \\ G(2,3 ; 6) & \text { if } m \equiv 0 & (\bmod 4)\end{array}\right.$

4. $G[3,3, m ; 4] \cong\left\{\begin{array}{lll}A_{4} & \text { if } m \equiv 1,5 & (\bmod 6) \\ \mathbf{S L}\left(2, \mathbf{Z}_{3}\right) & \text { if } m \equiv 2,4 & (\bmod 6) \\ \left(\mathbf{Z}_{3} \times \mathbf{Z}_{3}\right) \underset{\alpha_{41}}{ }<\left(\mathbf{Z}_{2} \times \mathbf{Z}_{2}\right) & \text { if } m \equiv 3 & (\bmod 6) \\ G(3,3 ; 4) & \text { if } m \equiv 0 & (\bmod 6)\end{array}\right.$ 
5. $G[2,3, m ; 8] \cong\left\{\begin{array}{lll}S_{4} & \text { if } m \equiv 1,5 & (\bmod 6) \\ \mathbf{Z}_{2} \underset{\alpha_{51}}{ } \mathbf{S L}\left(2, \mathbf{Z}_{3}\right) & \text { if } m \equiv 2,4 & (\bmod 6) \\ \mathbf{Z}_{2} \underset{\beta_{52}}{<}\left(\mathbf{Z}_{3} \underset{\alpha_{52}}{\triangleright<} A_{4}\right) & \text { if } m \equiv 3 & (\bmod 6) \\ G(2,3 ; 8) & \text { if } m \equiv 0 & (\bmod 6)\end{array}\right.$

6. $G[2,4, m ; 6] \cong\left\{\begin{array}{lll}S_{4} & \text { if } m \equiv 1,3,5,7 & (\bmod 8) \\ \left(\mathbf{Z}_{2} \times \mathbf{Z}_{2}\right) \underset{\alpha_{61}}{\triangleright} A_{4} & \text { if } m \equiv 2,6 & (\bmod 8) \\ \mathbf{Z}_{2} \underset{\beta_{62}}{<}\left(\mathbf{Z}_{4} \underset{\alpha_{62}}{\triangleright} A_{4}\right) & \text { if } m \equiv 4 \\ G(2,4 ; 6) & \text { if } m \equiv 0 & (\bmod 8)\end{array}\right.$

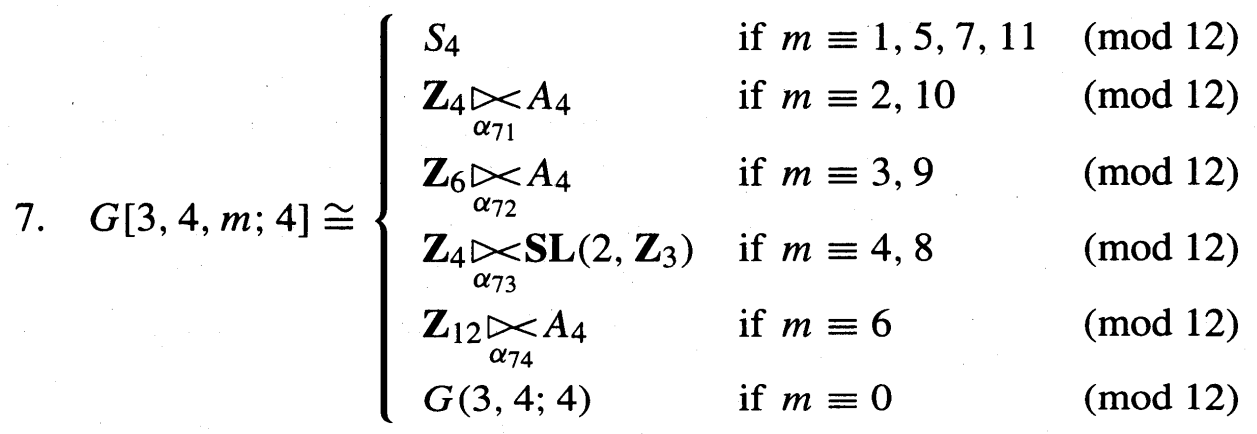

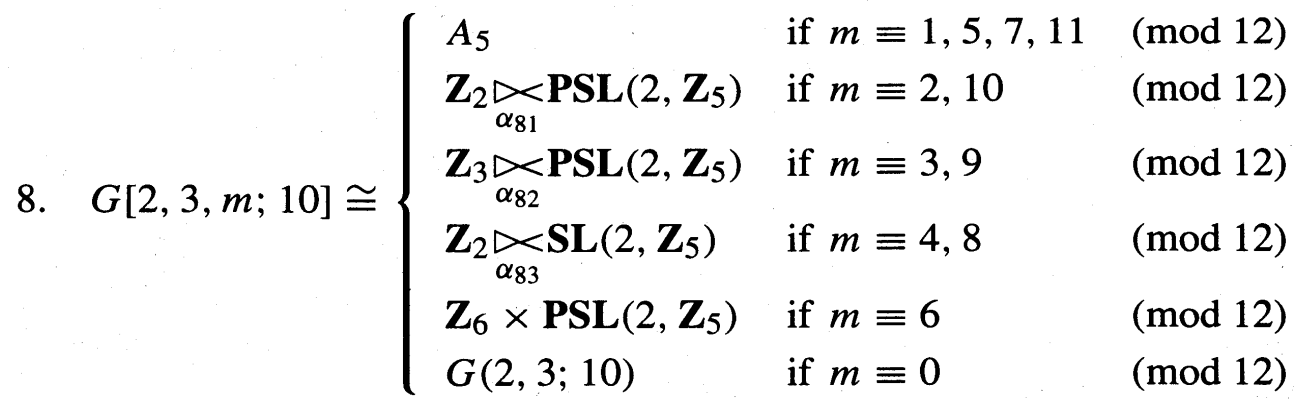

9. $G[2,5, m ; 6] \cong\left\{\begin{array}{lll}A_{5} & \text { if } m \equiv 1,3,7,9,11,13,17,19 & (\bmod 20) \\ \mathbf{Z}_{2} \underset{\alpha_{91}}{ } \operatorname{PSL}\left(2, \mathbf{Z}_{5}\right) & \text { if } m \equiv 2,6,14,18 & (\bmod 20) \\ \mathbf{Z}_{2} \underset{\alpha_{92}}{ } \operatorname{SL}\left(2, \mathbf{Z}_{5}\right) & \text { if } m \equiv 4,8,12,16 & (\bmod 20) \\ \mathbf{Z}_{5} \triangleright \operatorname{PSL}_{\alpha 93}\left(2, \mathbf{Z}_{5}\right) & \text { if } m \equiv 5,15 & (\bmod 20) \\ \mathbf{Z}_{10} \times \mathbf{P S L}\left(2, \mathbf{Z}_{5}\right) & \text { if } m \equiv 10 & (\bmod 20) \\ G(2,5 ; 6) & \text { if } m \equiv 0 & (\bmod 20)\end{array}\right.$ 


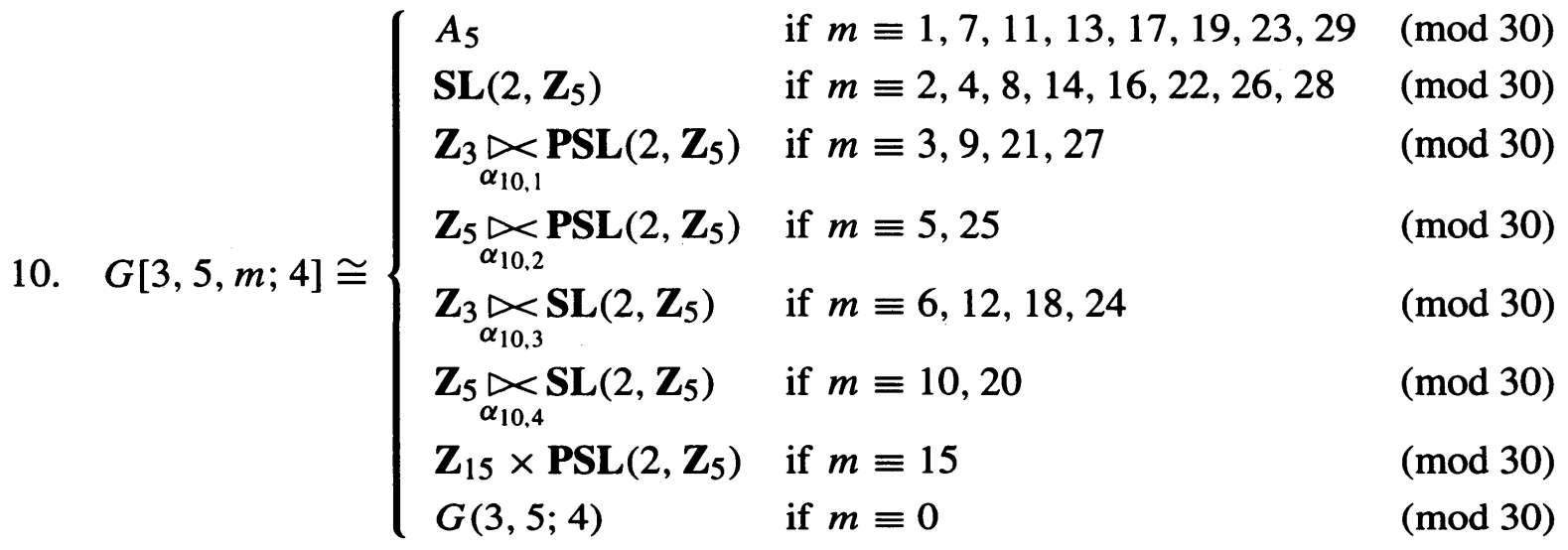

Proof. Since the proof of this proposition is similar to that of Proposition 3, we omit the details of the proof. However, we give the explicit descriptions of isomorphisms and actions so that we can calculate explicitly the orders of $a, b, c$ in $G\left[e_{1}, e_{2}, m ; q\right]$ (see Theorem 7 below). In the following, we write $G:=G\left[e_{1}, e_{2}, m ; q\right]$ for short.

(1) (i) Case where $m$ is odd. The isomorphism $G \cong D_{2 n_{1}}$ is given by $a \mapsto y$, $b \mapsto y^{-1} x, c \mapsto e$.

(ii) Case where $m$ is even. The isomorphism $G \cong D_{4 n_{1}}$ is given by $a \mapsto y, b \mapsto y^{-1} x$, $c \mapsto x^{n_{1}}$.

(2) The isomorphism $\left.G \cong \mathbf{Z}_{2} \underset{\alpha_{21}}{\not Z_{l}} \times \mathbf{Z}_{n_{2}}\right)$ is given by $a \mapsto(1,(0,0)), b \mapsto$ $(0,(0,1)), c \mapsto(0,(1,0))$. The action $\alpha_{21}: \mathbf{Z}_{2} \rightarrow \operatorname{Aut}\left(\mathbf{Z}_{l} \times \mathbf{Z}_{n_{2}}\right)$ is given by $\alpha_{21}(1)((1,0))=$ $(1,0), \alpha_{21}(1)((0,1))=(1,-1)$.

(3) (i) Case where $m \equiv 1,3(\bmod 4)$. The isomorphism $G \cong A_{4}$ is given by $a \mapsto$ (12)(34), $b \mapsto$ (234), $c \mapsto$ (1).

(ii) Case where $m \equiv 2(\bmod 4)$. The isomorphism $G \cong \mathbf{Z}_{2} \underset{\beta_{31}}{\bowtie}\left(\mathbf{Z}_{3} \underset{\alpha_{31}}{\triangleright}<\left(\mathbf{Z}_{2} \times \mathbf{Z}_{2}\right)\right)$ is given by $a \mapsto(1,(0,(0,0))), b \mapsto(0,(1,(0,0))), c \mapsto(1,(0,(1,1)))$. The action $\alpha_{31}: \mathbf{Z}_{3} \rightarrow \operatorname{Aut}\left(\mathbf{Z}_{2} \times \mathbf{Z}_{2}\right)$ is given by $\alpha_{31}(1)((1,0))=(0,1), \alpha_{31}(1)((0,1))=(1,1)$ and the action $\beta_{31}: \mathbf{Z}_{2} \rightarrow \operatorname{Aut}\left(\mathbf{Z}_{3} \bowtie \alpha_{31}<\left(\mathbf{Z}_{2} \times \mathbf{Z}_{2}\right)\right)$ is given by $\beta_{31}(1)((1,(0,0)))=(1,(0,1))$, $\beta_{31}(1)((0,(1,0)))=(0,(1,0)), \beta_{31}(1)((0,(0,1)))=(0,(0,1))$.

(iii) Case where $m \equiv 0(\bmod 4)$. The isomorphism $G \cong \mathbf{Z}_{2} \triangleright_{\beta_{3}}<\left(\mathbf{Z}_{3} \triangleright_{\alpha_{3}}<Q_{8}\right)$ is given by $\varphi_{3}$ in Proposition 3 since ord $\left((a b)^{3}\right)=4$ in $G(2,3 ; 6)$.

(4) (i) Case where $m \equiv 1,5(\bmod 6)$. The isomorphism $G \cong A_{4}$ is given by $a \mapsto$ (123), $b \mapsto$ (234), $c \mapsto$ (1).

(ii) Case where $m \equiv 2,4(\bmod 6)$. The isomorphism $G \cong \mathbf{S L}\left(2, \mathbf{Z}_{3}\right)$ is given by $a \mapsto\left[\begin{array}{ll}1 & 1 \\ 0 & 1\end{array}\right], b \mapsto\left[\begin{array}{ll}1 & 0 \\ 1 & 1\end{array}\right], c \mapsto 2 I_{2}$

(iii) Case where $m \equiv 3(\bmod 6)$. The isomorphism $G \cong\left(\mathbf{Z}_{3} \times \mathbf{Z}_{3}\right) \underset{\alpha_{41}}{\triangleright}\left(\mathbf{Z}_{2} \times \mathbf{Z}_{2}\right)$ is given by $a \mapsto((1,0),(0,0)), b \mapsto((0,1),(1,1)), c \mapsto((1,1),(0,0))$. The action $\alpha_{41}$ is given 
by $\alpha_{41}((1,0))((1,0))=(1,1), \alpha_{41}((0,1))((1,0))=(0,1), \alpha_{41}((1,0))((0,1))=(1,0)$, $\alpha_{41}((0,1))((0,1))=(1,1)$.

(iv) Case where $m \equiv 0(\bmod 6)$. The isomorphism $G \cong\left(\mathbf{Z}_{3} \times \mathbf{Z}_{3}\right) \underset{\alpha_{4}}{{ }_{\alpha}}<Q_{8}$ is given by $\varphi_{4}$ in Proposition 3 since ord $\left((a b)^{2}\right)=6$ in $G(3,3 ; 4)$.

(5) (i) Case where $m \equiv 1,5(\bmod 6)$. The isomorphism $G \cong S_{4}$ is given by $a \mapsto$ (34), $b \mapsto$ (123), $c \mapsto(1)$.

(ii) Case where $m \equiv 2,4(\bmod 6)$. Set $X=\left[\begin{array}{ll}1 & 1 \\ 0 & 1\end{array}\right], Y=\left[\begin{array}{ll}1 & 0 \\ 1 & 1\end{array}\right]$. The isomorphism $G \cong \mathbf{Z}_{2} \underset{\alpha_{51}}{\infty} \mathbf{S L}\left(2, \mathbf{Z}_{3}\right)$ is given by $a \mapsto\left(1, I_{2}\right), b \mapsto(0, Y), c \mapsto\left(0,2 I_{2}\right)$. The action $\alpha_{51}$ is given by $\alpha_{51}(1)(X)=Y, \alpha_{51}(1)(Y)=X$.

(iii) Case where $m \equiv 3(\bmod 6)$. Set $s=(234), t=(142) \in S_{4}$. The isomorphism $\left.G \cong \mathbf{Z}_{2} \underset{\beta_{52}}{\underset{\alpha_{52}}{\ltimes}}<\mathbf{Z}_{3} \underset{A_{4}}{ }\right)$ is given by $a \mapsto(1,(0,(1))), b \mapsto(0,(1,(1))), c \mapsto\left(0,\left(2, t^{2} s\right)\right)=$ $(0,(2,(123)))$. The action $\alpha_{52}: \mathbf{Z}_{3} \rightarrow \operatorname{Aut}\left(A_{4}\right)$ is given by $\alpha_{52}(1)(s)=t^{2}=(124)$, $\alpha_{52}(1)(t)=s t^{2}=(134)$. The action $\beta_{52}: \mathbf{Z}_{2} \rightarrow \operatorname{Aut}\left(\mathbf{Z}_{3} \underset{\alpha_{52}}{\prec}<A_{4}\right)$ is given by $\beta_{52}(1)(1,(1))=$ $(1, t), \beta_{52}(1)((0, s))=\left(0, s^{2}\right)=(0,(243)), \beta_{52}(1)((0, t))=\left(0, t^{2}\right)=(0,(124))$.

(iv) Case where $m \equiv 0(\bmod 6)$. The isomorphism $G \cong \mathbf{Z}_{2} \underset{\beta_{5}}{ }<\left(\mathbf{Z}_{3} \triangleright_{\alpha_{5}}<\mathbf{S L}\left(2, \mathbf{Z}_{3}\right)\right)$ is given by $\varphi_{5}$ in Proposition 3 since ord $\left((a b)^{4}\right)=6$ in $G(2,3 ; 8)$.

(6) (i) Case where $m \equiv 1,3,5,7(\bmod 8)$. The isomorphism $G \cong S_{4}$ is given by $a \mapsto$ (34), $b \mapsto(123), c \mapsto$ (1).

(ii) Case where $m \equiv 2,6(\bmod 8)$. Set $s=(234), t=(12)(34) \in S_{4}$. The isomorphism $G \cong\left(\mathbf{Z}_{2} \times \mathbf{Z}_{2}\right) \underset{\alpha_{61}}{\triangleright}<A_{4}$ is given by $a \mapsto((1,0),(1)), b \mapsto\left((0,1), s^{2} t s^{2}\right)=$ $((0,1),(143)), c \mapsto((1,1),(1))$. The action $\alpha_{61}: \mathbf{Z}_{2} \times \mathbf{Z}_{2} \rightarrow \operatorname{Aut}\left(A_{4}\right)$ is given by $\alpha_{61}((1,0))(s)=s^{2}=(243), \alpha_{61}((0,1))(s)=s^{2}=(243), \alpha_{61}((1,0))(t)=s^{2} t s=$ (14) $(23), \alpha_{61}((0,1))(t)=s^{2} t s=(14)(23)$.

(iii) Case where $m \equiv 4(\bmod 8)$. The isomorphism $G \cong \mathbf{Z}_{2} \underset{\beta_{62}}{\triangleright}<\left(\mathbf{Z}_{4} \underset{\alpha_{62}}{\triangleright}<A_{4}\right)$ is given by $a \mapsto(1,(0,(1))), b \mapsto(0,(1,(1))), c \mapsto\left(1,\left(1,(s t)^{2}\right)\right)=(1,(1,(132)))$. The action $\alpha_{62}: \mathbf{Z}_{4} \rightarrow \operatorname{Aut}\left(A_{4}\right)$ is given by $\alpha_{62}(1)(s)=s t s=(134), \alpha_{62}(1)(t)=s^{2} t s=(14)(23)$ and the action $\beta_{62}: \mathbf{Z}_{2} \rightarrow \operatorname{Aut}\left(\mathbf{Z}_{4}{\underset{\alpha}{62}}_{<}<A_{4}\right)$ is given by $\beta_{62}(1)(1,(1))=\left(1, s^{2} t s^{2}\right)=(1,(143))$, $\beta_{62}(1)((0, s))=\left(0, s^{2}\right)=(0,(243)), \beta_{62}(1)((0, t))=(0, t)$.

(iv) Case where $m \equiv 0(\bmod 8)$. The isomorphism $G \cong \mathbf{Z}_{2} \triangleright_{\beta_{6}}<\left(\mathbf{Z}_{4} \triangleright_{\alpha_{6}}<\mathbf{S L}\left(2, \mathbf{Z}_{3}\right)\right)$ is given by $\varphi_{6}$ in Proposition 3 since ord $\left((a b)^{3}\right)=8$ in $G(2,4 ; 6)$.

(7) (i) Case where $m \equiv 1,5,7,11(\bmod 12)$. The isomorphism $G \cong S_{4}$ is given by $a \mapsto(123), b \mapsto$ (4321), $c \mapsto$ (1).

(ii) Case where $m \equiv 2,10(\bmod 12)$. Set $s=(234), t=(142) \in S_{4}$. The isomorphism $G \cong \mathbf{Z}_{4} \triangleright \alpha_{71}<A_{4}$ is given by $a \mapsto(0, s), b \mapsto(1,(1)), c \mapsto(2, s t)=(2,(12)(34))$. The action $\alpha_{71}: \mathbf{Z}_{4} \rightarrow \operatorname{Aut}\left(A_{4}\right)$ is given by $\alpha_{71}(1)(s)=t^{2} s=(123), \alpha_{71}(1)(t)=s$. 
(iii) Case where $m \equiv 3,9(\bmod 12)$. Set $s=(234), t=(12)(34) \in S_{4}$. The isomorphism $G \cong \mathrm{Z}_{6} \underset{\alpha_{72}}{\triangleright}<A_{4}$ is given by $a \mapsto(4, s t)=(4,(132)), b \mapsto(3, t s t)=(3,(143))$, $c \mapsto(4,(1))$. The action $\alpha_{72}: \mathbf{Z}_{6} \rightarrow \operatorname{Aut}\left(A_{4}\right)$ is given by $\alpha_{72}(1)(s)=s^{2}=(243)$, $\alpha_{72}(1)(t)=s^{2} t s=(14)(23)$.

(iv) Case where $m \equiv 4,8(\bmod 12) . \operatorname{Sex} X=\left[\begin{array}{ll}1 & 1 \\ 0 & 1\end{array}\right], Y=\left[\begin{array}{ll}1 & 0 \\ 1 & 1\end{array}\right] \in \mathbf{S L}\left(2, \mathbf{Z}_{3}\right)$. The isomorphism $G \cong \mathbf{Z}_{4} \underset{\alpha_{73}}{\triangleright} \mathbf{S L}\left(2, \mathbf{Z}_{3}\right)$ is given by $a \mapsto(0, X), b \mapsto\left(1, I_{2}\right), c \mapsto\left(2,(X Y)^{3}\right)$ $=\left(2,\left[\begin{array}{ll}1 & 2 \\ 2 & 2\end{array}\right]\right)$. The action $\alpha_{73}: \mathbf{Z}_{4} \rightarrow \operatorname{Aut}\left(\mathbf{S L}\left(2, \mathbf{Z}_{3}\right)\right)$ is given by $\alpha_{73}(1)(X)=X Y X^{-1}$ $=\left[\begin{array}{ll}2 & 2 \\ 1 & 0\end{array}\right], \alpha_{73}(1)(Y)=X$.

(v) Case where $m \equiv 6(\bmod 12)$. Set $s=(234), t=(12)(34) \in S_{4}$. The isomorphism $G \cong \mathrm{Z}_{12} \underset{\alpha_{74}}{<} A_{4}$ is given by $a \mapsto(4, t s)=(4,(124)), b \mapsto(9, s t)=(9,(132)), c \mapsto$ $(10,(1))$. The action $\alpha_{74}: \mathbf{Z}_{12} \rightarrow \operatorname{Aut}\left(A_{4}\right)$ is given by $\alpha_{74}(1)(s)=s^{2}=(243), \alpha_{74}(1)(t)=$ $t$.

(vi) Case where $m \equiv 0(\bmod 12)$. The isomorphism $G \cong \mathbf{Z}_{12} \underset{\alpha_{7}}{\triangleright}<\mathbf{S L}\left(2, \mathbf{Z}_{3}\right)$ is given by $\varphi_{7}$ in Proposition 3 since ord $\left((a b)^{2}\right)=12$ in $G(3,4 ; 4)$.

(8) (i) Case where $m \equiv 1,5,7,11(\bmod 12)$. The isomorphism $G \cong A_{5}$ is given by $a \mapsto(12)(45), b \mapsto(134), c \mapsto(1)$.

(ii) Case where $m \equiv 2,10(\bmod 12)$. Set $X=\left[\begin{array}{ll}0 & 1 \\ 4 & 1\end{array}\right], Y=\left[\begin{array}{ll}0 & 1 \\ 4 & 4\end{array}\right] \in \operatorname{PSL}\left(2, \mathbf{Z}_{5}\right)$. The isomorphism $G \cong \mathbf{Z}_{2} \underset{\alpha_{81}}{\triangleright} \operatorname{PSL}\left(2, \mathbf{Z}_{5}\right)$ is given by $a \mapsto\left(1, I_{2}\right), b \mapsto(0, X), c \mapsto$ $(2, Y X Y X Y)=\left(2,\left[\begin{array}{ll}3 & 0 \\ 0 & 2\end{array}\right]\right)$. The action $\alpha_{81}: \mathbf{Z}_{2} \rightarrow \operatorname{Aut}\left(\operatorname{PSL}\left(2, \mathbf{Z}_{5}\right)\right)$ is given by $\alpha_{81}(1)(X)=Y, \alpha_{81}(1)(Y)=X$.

(iii) Case where $m \equiv 3,9(\bmod 12)$. Set $X=\left[\begin{array}{ll}0 & 1 \\ 4 & 0\end{array}\right], Y=\left[\begin{array}{ll}1 & 1 \\ 3 & 4\end{array}\right], Z=\left[\begin{array}{ll}1 & 2 \\ 4 & 4\end{array}\right] \in$ $\operatorname{PSL}\left(2, \mathbf{Z}_{5}\right)$. The isomorphism $G \cong \mathbf{Z}_{3} \underset{\alpha_{82}}{\prec} \operatorname{PSL}\left(2, \mathbf{Z}_{5}\right)$ is given by $a \mapsto(0, X), b \stackrel{\mapsto}{\mapsto}\left(1, I_{2}\right)$, $c \mapsto(1, Z X Y Z X Y Z X Y Z)=\left(1,\left[\begin{array}{ll}1 & 1 \\ 4 & 0\end{array}\right]\right)$. The action $\alpha_{82}: \mathbf{Z}_{3} \rightarrow \operatorname{Aut}\left(\operatorname{PSL}\left(2, \mathbf{Z}_{5}\right)\right)$ is given by $\alpha_{82}(1)(X)=Z, \alpha_{82}(1)(Y)=X, \alpha_{82}(1)(Z)=Y$.

(iv) Case where $m \equiv 4,8(\bmod 12)$. Set $X=\left[\begin{array}{ll}0 & 1 \\ 4 & 4\end{array}\right], Y=\left[\begin{array}{ll}0 & 4 \\ 1 & 4\end{array}\right] \in \mathbf{S L}\left(2, \mathbf{Z}_{5}\right)$. The isomorphism $G \cong \mathbf{Z}_{2} \underset{\alpha_{83}}{\triangleright} \mathbf{S L}\left(2, \mathbf{Z}_{5}\right)$ is given by $a \mapsto\left(1, I_{2}\right), b \mapsto(0, X), c \mapsto\left(1,(X Y)^{7} X\right)$ 
$=\left(1,\left[\begin{array}{ll}2 & 0 \\ 0 & 3\end{array}\right]\right) . \quad$ The action $\alpha_{83}: \mathbf{Z}_{2} \rightarrow \operatorname{Aut}\left(\mathbf{S L}\left(2, \mathbf{Z}_{5}\right)\right)$ is given by $\alpha_{83}(1)(X)=Y$, $\alpha_{83}(1)(Y)=X$.

(v) Case when $m \equiv 6(\bmod 12)$. Set $X=\left[\begin{array}{ll}1 & 0 \\ 1 & 1\end{array}\right], Y=\left[\begin{array}{ll}2 & 1 \\ 1 & 1\end{array}\right] \in \operatorname{PSL}\left(2, \mathbf{Z}_{5}\right)$. The isomorphism $G \cong \mathbf{Z}_{6} \times \operatorname{PSL}\left(2, \mathbf{Z}_{5}\right)$ is given by $a \mapsto\left(3, X Y^{3} X^{3} Y\right)=\left(3,\left[\begin{array}{ll}2 & 0 \\ 3 & 3\end{array}\right]\right)$, $b \mapsto\left(4, Y^{3} X^{2}\right)=\left(4,\left[\begin{array}{ll}4 & 3 \\ 3 & 0\end{array}\right]\right), c \mapsto\left(5, I_{2}\right)$

(vi) Case where $m \equiv 0(\bmod 12)$. The isomorphism $G \cong \mathbf{Z}_{6} \triangleright_{\alpha_{8}}<\mathbf{S L}\left(2, \mathbf{Z}_{5}\right)$ is given by $\varphi_{8}$ is Proposition 3 since ord $\left((a b)^{5}\right)=12$ in $G(2,3 ; 10)$.

(9) (i) Case where $m \equiv 1,3,5,7,9,11,13,17,19(\bmod 20)$. The isomorphism $G \cong$ $A_{5}$ is given by $a \mapsto(12)(45), b \mapsto(13542), c \mapsto(1)$.

(ii) Case where $m \equiv 2,6,14,18(\bmod 20)$. Set $X=\left[\begin{array}{ll}1 & 0 \\ 1 & 1\end{array}\right], Y=\left[\begin{array}{ll}0 & 4 \\ 1 & 2\end{array}\right] \epsilon$ $\operatorname{PSL}\left(2, \mathbf{Z}_{5}\right)$. The isomorphism $G \cong \mathbf{Z}_{2} \underset{\alpha_{91}}{\triangleright} \operatorname{PSL}\left(2, \mathbf{Z}_{5}\right)$ is given by $a \mapsto\left(1, I_{2}\right), b \mapsto(0, X)$, $c \mapsto(1, X Y X)=\left(1,\left[\begin{array}{ll}4 & 4 \\ 2 & 1\end{array}\right]\right)$. The action $\alpha_{91}: \mathbf{Z}_{2} \rightarrow \operatorname{Aut}\left(\operatorname{PSL}\left(2, \mathbf{Z}_{5}\right)\right)$ is given by $\alpha_{91}(1)(X)=Y, \alpha_{91}(1)(Y)=X$.

(iii) Case where $m \equiv 4,8,12,16(\bmod 20)$. Set $X=\left[\begin{array}{ll}1 & 0 \\ 1 & 1\end{array}\right], Y=\left[\begin{array}{ll}1 & 4 \\ 0 & 1\end{array}\right] \epsilon$ $\mathbf{S L}\left(2, \mathbf{Z}_{5}\right)$. The isomorphism $G \cong \mathbf{Z}_{2} \underset{\alpha_{92}}{\triangleright}<\mathbf{S L}\left(2, \mathbf{Z}_{5}\right)$ is given by $a \mapsto\left(1, I_{2}\right), b \stackrel{\mapsto}{\mapsto}(0, X)$, $c \mapsto\left(1,(X Y X)^{3}\right)=\left(1,\left[\begin{array}{ll}0 & 1 \\ 4 & 0\end{array}\right]\right)$. The action $\alpha_{92}: \mathbf{Z}_{2} \rightarrow \operatorname{Aut}\left(\mathbf{S L}\left(2, \mathbf{Z}_{5}\right)\right)$ is given by $\alpha_{92}(1)(X)=Y, \alpha_{92}(1)(Y) \stackrel{=}{=}$.

(iv) Case where $m \equiv 5,15(\bmod 20)$. Set $X=\left[\begin{array}{ll}1 & 1 \\ 3 & 4\end{array}\right], Y=\left[\begin{array}{ll}0 & 1 \\ 4 & 0\end{array}\right], Z=\left[\begin{array}{ll}1 & 4 \\ 2 & 4\end{array}\right] \epsilon$ $\operatorname{PSL}\left(2, \mathbf{Z}_{5}\right)$. This isomorphism $G \cong \mathbf{Z}_{5} \underset{\alpha_{93}}{\bowtie} \mathbf{P S L}\left(2, \mathbf{Z}_{5}\right)$ is given by $a \mapsto(0, X), b \stackrel{\mapsto}{\mapsto}\left(1, I_{2}\right)$, $c \mapsto\left(2,(X Y Z)^{4}\right)=\left(1,\left[\begin{array}{ll}1 & 0 \\ 3 & 1\end{array}\right]\right)$. The action $\alpha_{93}: \mathbf{Z}_{5} \rightarrow \operatorname{Aut}\left(\operatorname{PSL}\left(2, \mathbf{Z}_{5}\right)\right)$ is given by $\alpha_{93}(1)(X)=X Y Z Y X=\left[\begin{array}{ll}3 & 4 \\ 0 & 2\end{array}\right], \alpha_{93}(1)(Y)=X, \alpha_{93}(1)(Z)=Y$. 
(v) Case where $m \equiv 10(\bmod 20)$. Set $X=\left[\begin{array}{ll}1 & 0 \\ 1 & 1\end{array}\right], Y=\left[\begin{array}{ll}1 & 1 \\ 0 & 1\end{array}\right] \in \mathbf{P S L}\left(2, \mathbf{Z}_{5}\right)$. The isomorphism $G \cong \mathbf{Z}_{10} \times \operatorname{PSL}\left(2, \mathbf{Z}_{5}\right)$ is given by $a \mapsto\left(5, Y^{2} X Y X^{2}\right)=\left(5,\left[\begin{array}{ll}3 & 0 \\ 0 & 2\end{array}\right]\right)$, $b \mapsto\left(6, X^{-1} Y^{-1} X\right)=\left(6,\left[\begin{array}{ll}0 & 4 \\ 1 & 2\end{array}\right]\right), c \mapsto\left(7, I_{2}\right)$.

(vi) Case where $m \equiv 0(\bmod 20)$. The isomorphism $G \cong \mathbf{Z}_{10} \underset{\alpha_{9}}{\bowtie L} \mathbf{S L}\left(2, \mathbf{Z}_{5}\right)$ is given by $\varphi_{9}$ in Theorem 9 since ord $\left((a b)^{3}\right)=20$ in $G(2,5 ; 6)$.

(10) (i) Case where $m \equiv 1,7,11,13,17,19,23,29(\bmod 30)$. The isomorphism $G \cong A_{5}$ is given by $a \mapsto(134), b \mapsto$ (12)(45), $c \mapsto(1)$.

(ii) Case where $m \equiv 2,4,8,14,16,22,26,28(\bmod 30)$. Set $X=\left[\begin{array}{ll}0 & 1 \\ 4 & 4\end{array}\right], Y=$ $\left[\begin{array}{ll}1 & 0 \\ 1 & 1\end{array}\right] \in \mathbf{S L}\left(2, \mathbf{Z}_{5}\right)$. The isomorphism $G \cong \mathbf{S L}\left(2, \mathbf{Z}_{5}\right)$ is given by $a \mapsto X, b \mapsto Y$,
$c \mapsto 4 I_{2}$.

(iii) Case where $m \equiv 3,9,21,27(\bmod 30)$. Set $X=\left[\begin{array}{ll}1 & 0 \\ 1 & 1\end{array}\right], Y=\left[\begin{array}{ll}0 & 4 \\ 1 & 2\end{array}\right] \epsilon$ $\operatorname{PSL}\left(2, \mathbf{Z}_{5}\right)$. The isomorphism $G \cong \mathbf{Z}_{2} \underset{\alpha_{10,1}}{\triangleright} \mathbf{P S L}\left(2, \mathbf{Z}_{5}\right)$ is given by $a \mapsto\left(1, I_{2}\right), b \mapsto(10, X)$, $c \mapsto\left(1,(X Y)^{2}\right)$. The action $\alpha_{10,1}: \mathbf{Z}_{3} \rightarrow \operatorname{Aut}\left(\operatorname{PSL}\left(2, \mathbf{Z}_{5}\right)\right)$ is given by $\alpha_{10,1}(1)(X)=$ $X Y X^{-1}=\left[\begin{array}{ll}1 & 4 \\ 0 & 1\end{array}\right], \alpha_{10,1}(1)(Y)=X$.

(iv) Case where $m \equiv 5,25(\bmod 30)$. Set $X=\left[\begin{array}{ll}1 & 1 \\ 2 & 3\end{array}\right], Y=\left[\begin{array}{ll}1 & 1 \\ 4 & 0\end{array}\right] \in \mathbf{P S L}\left(2, \mathbf{Z}_{5}\right)$. The isomorphism $G \cong \mathbf{Z}_{5} \underset{\alpha_{10.2}}{\propto} \operatorname{PSL}\left(2, \mathbf{Z}_{5}\right)$ is given by $a \mapsto(0, X), \quad b \mapsto\left(1, I_{2}\right)$, $c \mapsto\left(3,(X Y)^{4}\right)=\left(3,\left[\begin{array}{ll}2 & 4 \\ 1 & 0\end{array}\right]\right)$. The action $\alpha_{10,2}: \mathbf{Z}_{5} \rightarrow \operatorname{Aut}\left(\mathbf{P S L}\left(2, \mathbf{Z}_{5}\right)\right)$ is given by $\alpha_{10,2}(1)(X)=X Y X^{-1}=\left[\begin{array}{ll}3 & 1 \\ 3 & 3\end{array}\right], \alpha_{10,2}(1)(Y)=X$.

(v) Case where $m \equiv 6,12,18,24(\bmod 30)$. Set $X=\left[\begin{array}{ll}1 & 0 \\ 1 & 1\end{array}\right], Y=\left[\begin{array}{ll}1 & 4 \\ 0 & 1\end{array}\right] \epsilon$ $\mathbf{S L}\left(2, \mathbf{Z}_{5}\right)$. The isomorphism $G \cong \mathbf{Z}_{3} \underset{\alpha_{10,3}}{\triangleright} \mathbf{S L}\left(2, \mathbf{Z}_{5}\right)$ is given by $a \mapsto\left(1, I_{2}\right), b \mapsto(0, X)$, $c \mapsto\left(1,(X Y)^{5}\right)=\left(1,\left[\begin{array}{ll}0 & 1 \\ 4 & 1\end{array}\right]\right)$. The action $\alpha_{10,3}: \mathbf{Z}_{3} \rightarrow \operatorname{Aut}\left(\mathbf{S L}\left(2, \mathbf{Z}_{5}\right)\right)$ is given by $\alpha_{10,3}(1)(X)=X Y X^{-1}=\left[\begin{array}{ll}2 & 4 \\ 1 & 0\end{array}\right], \alpha_{10,3}(1)(Y)=X$. 
(vi) Case where $m \equiv 10,20(\bmod 30)$. Set $X=\left[\begin{array}{ll}0 & 1 \\ 4 & 4\end{array}\right], Y=\left[\begin{array}{ll}0 & 4 \\ 1 & 4\end{array}\right] \in \mathbf{S L}\left(2, \mathbf{Z}_{5}\right)$. The isomorphism $G \cong \mathbf{Z}_{5} \bowtie<\mathbf{S L}\left(2, \mathbf{Z}_{5}\right)$ is given by $a \mapsto(0, X), \quad b \mapsto\left(1, I_{2}\right)$, $c \mapsto\left(3,(X Y)^{9}\right)=\left(3,\left[\begin{array}{ll}2 & 1 \\ 1 & 1\end{array}\right]\right) . \quad$ The action $\alpha_{10,4}: \mathbf{Z}_{5} \rightarrow \operatorname{Aut}\left(\mathbf{S L}\left(2, \mathbf{Z}_{5}\right)\right)$ is given by $\alpha_{10,4}(1)(X)=X Y X^{-1}=\left[\begin{array}{ll}3 & 4 \\ 3 & 1\end{array}\right], \alpha_{10,4}(1)(Y)=X$.

(vii) Case where $m \equiv 15(\bmod 30)$. Set $X=\left[\begin{array}{ll}1 & 0 \\ 1 & 1\end{array}\right], Y=\left[\begin{array}{ll}1 & 1 \\ 0 & 1\end{array}\right] \in \operatorname{PSL}\left(2, \mathbf{Z}_{5}\right)$. The isomorphism $G \cong \mathbf{Z}_{15} \times \mathbf{P S L}\left(2, \mathbf{Z}_{5}\right)$ is given by $a \mapsto\left(10, Y^{-1} X^{-1} Y^{2} X^{2} Y^{-2} X^{-2}\right)=$ $\left(10,\left[\begin{array}{ll}4 & 2 \\ 2 & 0\end{array}\right]\right), b \mapsto\left(6, X^{-1} Y^{-1} X Y\right)=\left(6,\left[\begin{array}{ll}0 & 4 \\ 1 & 3\end{array}\right]\right), c \mapsto\left(13, I_{2}\right)$.

(viii) Case where $m \equiv 0(\bmod 30)$. The isomorphism $G \cong \mathbf{Z}_{15} \underset{\alpha_{10}}{\triangleright}<\mathbf{S L}\left(2, \mathbf{Z}_{5}\right)$ is given by $\varphi_{10}$ in Proposition 3 since ord $\left((a b)^{2}\right)=30$ in $G(3,5 ; 4)$.

From Proposition 6, we get the following geometric result, which is our second main result.

THEOREM 7. (1) Let the triple $\left(e_{1}, e_{2}, q\right)$ be as in Proposition 6. Then the necessary and sufficient condition for the existence of the maximal Galois covering of $\left(\mathbf{P}^{2}, e_{1} \overline{B_{q}^{1}}+e_{2} \overline{B_{q}^{2}}+\right.$ $\left.m l_{\infty}\right)$ is as follows:

1. In the case $\left(e_{1}, e_{2}, q\right)=\left(2,2,2 n_{1}\right), m=1,2$.

2. In the case $\left(e_{1}, e_{2}, q\right)=\left(2, n_{2}, 4\right), m=$ any positive divisor of $n_{2}$.

3. In the case $\left(e_{1}, e_{2}, q\right)=(2,3,6), m=1,2,4$.

4. In the case $\left(e_{1}, e_{2}, q\right)=(3,3,4), m=1,2,3,6$.

5. In the case $\left(e_{1}, e_{2}, q\right)=(2,3,8), m=1,2,3,6$.

6. In the case $\left(e_{1}, e_{2}, q\right)=(2,4,6), m=1,2,4,8$.

7. In the case $\left(e_{1}, e_{2}, q\right)=(3,4,4), m=1,2,3,4,6,12$.

8. In the case $\left(e_{1}, e_{2}, q\right)=(2,3,10), m=1,2,3,4,6,12$.

9. In the case $\left(e_{1}, e_{2}, q\right)=(2,5,6), m=1,2,4,5,10,20$.

10. In the case $\left(e_{1}, e_{2}, q\right)=(3,5,4), m=1,2,3,5,6,10,15,30$.

(2) The maximal Galois coverings in (1) are all normal projective rational surfaces.

(3) Assume $m$ is the maximal value in each of the above 10 cases (for instance, $m=30$ in the case 10). Then the corresponding maximal Galois covering contains $\mathbf{A}^{2}$ as an open dense subset.

PROOF. (1) We check the case 1, the other cases being treated similarly. Suppose $m$ is odd. Then we have $\operatorname{ord}(a)=\operatorname{ord}(b)=2, \operatorname{ord}(c)=1$ by Proposition 6. Hence the maximal Galois covering exists if and only if $m=1$ by [NT, Corollary 1.5]. 
Suppose $m$ is even. Then we have $\operatorname{ord}(a)=\operatorname{ord}(b)=2, \operatorname{ord}(c)=2$ and the maximal Galois covering exists if and only if $m=2$ similarly.

(2) From the general construction of maximal Galois coverings (cf. [Na, Theorem 1.3.8]), our maximal Galois covering $X$ is a projectivization of a quotient of $\mathbf{A}^{2}$ by a finite group so that it is a unirational surface. Since $X$ is rational (cf. [B, Corollaire V.5]), and normal by definition, the assertion (2) is done.

(3) If $m$ is maximal, then $X$ is a projectiviazation of the quotient of $\mathbf{A}^{2}$ by the trivial group.

\section{References}

[B] A. BEAUVILle, Surfaces algébriques complexes, astérisque 54 (1978), Société Mathématique de France:

[CM] H. S. M. COXETER and W. O. J. MOSER, Generaters and relations for discrete groups (third edition), Springer (1972).

[J] D. L. JohnSon, Presentations of Groups, London Math. Soc. Student Texts 15, Cambridge Univ. Press (1990).

[K] T. KAMBAYASHI, Automorphism group of a polynomial ring and algebraic group actions on an affine space, J. Algebra 60 (1979), 439-451.

[Na] M. NAMBA, Branched coverings and algebraic functions, Pitman Research Notes in Math. Series 161, Longman (1987).

[Ni] H. Nishikubo, On some Galois groups of affine and projective planes, Master Thesis, Tokyo Denki Univ. (1997) (in Japanese).

[NT] T. NAKANO and K. TAMAI, On some maximal Galois coverings over affine and projective planes, Osaka J. Math. 33 (1996), 347-364.

[ST] G. C. SHEPHARD and J. A. TodD, Finite unitary reflection groups, Canad. J. Math. 6 (1954), 274-304.

[T] K. TAmaI, On the Galois coverings over $\mathbf{A}^{2}$ with branch locus $w^{2}=v^{q}$, Master Thesis, Tokyo Denki Univ. (1994) (in Japanese).

Present Address:

Department of Mathematical Sciences, College of Science ANd EngineERing, TOKYO DENKI UNIVERSITY,

HatOYAMA-MACHI, Hiki-gUN, SAITAMA, 350-0394 JAPAN.

e-mail:nakano@r.dendai.ac.jp 